\title{
SINGLY PERIODIC SOLUTIONS OF THE ALLEN-CAHN EQUATION AND THE TODA LATTICE
}

\author{
MICHA£ KOWALCZYK, YONG LIU, AND JUNCHENG WEI
}

\begin{abstract}
The Allen-Cahn equation $-\Delta u=u-u^{3}$ in $\mathbb{R}^{2}$ has family of trivial singly periodic solutions that come from the one dimensional periodic solutions of the problem $-u^{\prime \prime}=u-u^{3}$. In this paper we construct a non-trivial family of singly periodic solutions to the Allen-Cahn equation. Our construction relies on the connection between this equation and the infinite Toda lattice. We show that for each one-soliton solution to the infinite Toda lattice we can find a singly periodic solution to that Allen-Cahn equation, such that its level set is close to the scaled one-soliton.
\end{abstract}

\section{INTRODUCTION}

In this paper we construct new entire solutions of the Allen-Cahn equation in the plane:

$$
-\Delta u=u-u^{3} \text { in } \mathbb{R}^{2} \text {. }
$$

The monotone bounded solutions of this equation have been extensively studied in the literature. These are called planar solutions and they are given by $H\left(\mathrm{e}^{\perp} \cdot \mathrm{x}-r\right)$, where $\mathrm{e}$ is a unit vector in $\mathbb{R}^{2}, \mathrm{e}^{\perp}=e^{i \pi / 2} \mathrm{e}$, $r \in \mathbb{R}$ is a constant, and $H(s)=\tanh \frac{s}{\sqrt{2}}$ is the unique odd, heteroclinic solution:

$$
-H^{\prime \prime}=H-H^{3}, \quad H( \pm \infty)= \pm 1
$$

The planar solutions are special examples of the so-called multiple-end solutions, which by definition are solutions whose nodal curves are asymptotic at infinity to finitely many copies of half planar solutions (see [5], [4], [13], [14], [15], [16] where the subject was treated extensively). Any planar solution has an important property of being monotone in one direction. According to the De Giorgi conjecture any bounded solution to (1.1) which is monotone in one direction must be a planar solution [11] (see also [2], [20], [6] for higher dimensional results). In general, mulitple-end solutions will not be monotone in any direction, but it turns out that they represent an important class of entire solutions.

Using infinite dimensional Lyapunov-Schmidt reduction and perturbation method, for each integer $n>1$, a family of $2 n$-end solutions is constructed in [5]. Their nodal curves are close to solutions to the classical Toda system:

$$
c_{*} q_{i}^{\prime \prime}=e^{\sqrt{2}\left(q_{i-1}-q_{i}\right)}-e^{\sqrt{2}\left(q_{i}-q_{i+1}\right)}, \quad 1 \leq i \leq n,
$$

where $q_{0}=-\infty$ and $q_{n+1}=+\infty$, and $c_{*}$ is a fixed constant. The Toda system is a classical example of an integrable system describing the dynamics of $n$ particles moving along a straight line and whose interaction with their closest neighbour is given by the exponential of the negative of their distance. Another construction of $2 n$-end solutions was given in [16] (see also [1], [3]) .

A particular example of $2 n$ solutions is the set of four-end solutions. It contains the so called saddle solution, whose nodal set consists of two straight line intersecting at the right angle. It is shown in [15] and [14] that all four-end solutions are continuous deformations of the saddle solution. This gives a classification of the set of four-end solutions. On the other hand when the number of ends is bigger than four, no satisfactory classification result is available up to now, but it is known [15] that any multiple-end solutions must have

1991 Mathematics Subject Classification. 35J61.

Y. Liu is partially supported by NSFC grant 11101141 and the Fundamental Research Funds for the Central Universities 13MS39. M. Kowalczyk is partially supported by Chilean research grants Fondecyt 1130126 and Fondo Basal CMM-Chile, and part of this work was done during his stay in the École Polytechnique. J. Wei is partially supported by NSERC of Canada. We would like to thank Professor Frank Pacard for the discussion we had during the preparation of this paper. 
finite Morse index. We also expect that any finite Morse index solution to be multiple-ended (this is proven in [12] under an extra assumption on the growth of the energy). The purpose of this paper is to construct non-trivial (i.e. depending on two spacial variables) solutions of (1.1) with infinitely many ends and infinite Morse index.

In the one dimensional case, the Allen-Cahn equation reduces to a second order ODE. It is easy to see that besides the heteroclinc solution, this ODE also admits periodic solutions, which in fact could be expressed as a suitable scaling of the Jacobi elliptic functions. Clearly, they could be lifted to $\mathbb{R}^{2}$, yielding periodic solutions depending only on one space variable. Since they are periodic, their Morse index is infinite but because they depend on just one variable they are at the same time one-dimensional. Naturally, one would like to find non-trivial periodic solutions. In this respect, a bifurcation analysis has been carried out in [21] and some nontrivial doubly periodic solutions were found.

In this paper we will construct new entire solutions of (1.1) in $\mathbb{R}^{2}$ which are singly periodic. As we will see, the nodal set of the solutions we construct will be determined by the solutions of the infinite Toda lattice:

$$
c_{*} q_{i}^{\prime \prime}=e^{\sqrt{2}\left(q_{i-1}-q_{i}\right)}-e^{\sqrt{2}\left(q_{i}-q_{i+1}\right)}, \quad i \in \mathbb{Z} .
$$

Due to the fact that the number of particles, whose positions are given by the functions $q_{i}, i \in \mathbb{Z}$, is infinite this system is more complicated than the classical Toda system, however it is still integrable. For us a special solution called one-soliton will be of crucial importance and we will describe it now. Given a parameter $k>0$, let us denote

$$
c=c(k):=\frac{\sinh k}{k}>1
$$

The function

$$
S_{c}(t):=\ln \frac{\cosh k\left(t-\frac{1}{2}\right)}{\cosh k\left(t+\frac{1}{2}\right)}
$$

is odd and satisfies, for $t$ large,

$$
\left|S_{c}(t)+k\right| \leq C e^{-2 k t}
$$

Direct computation shows that

$$
s_{i}(t ; c, \lambda):=S_{c}(i-c \lambda t)-2 i \ln \lambda, \quad i \in \mathbb{Z}, \quad \lambda>0,
$$

solve the Toda lattice equation:

$$
s_{i}^{\prime \prime}=e^{s_{i-1}-s_{i}}-e^{s_{i}-s_{i+1}}, \quad i \in \mathbb{Z} .
$$

This one soliton solution and its properties were studied in a series of papers by Friesecke and Pego [7], [8], [9], [10] and Mizumachi and Pego [18].

Rescaling the Toda lattice equation we see that:

$$
q_{i}=q_{i}(t ; c, \varepsilon):=\frac{1}{\sqrt{2}} s_{i}\left(\sqrt{\sqrt{2} c_{*}^{-1}} t ; c, \varepsilon\right), \quad i \in \mathbb{Z},
$$

is a family of one-soliton solutions of (1.3). The distance $q_{i}-q_{i-1}$ between two adjacent "particles" is approximately $\sqrt{2}|\ln \varepsilon|$, which means they are far away from each other if the parameter $\varepsilon$ is small.

Now we can state our main result precisely.

Theorem 1.1. For each sufficiently small $\varepsilon>0$ and for each $c>0$ the Allen-Cahn equation (1.1) has a non-trivial singly periodic solution $u_{c, \varepsilon}$ with minimal period $2 \mathrm{e}$, where

$$
\mathrm{e}=\left(\frac{1}{\varepsilon \sqrt{\sqrt{2} c_{*}^{-1}}}, \sqrt{2}|\ln \varepsilon|\right)
$$

Moreover, it holds

$$
\begin{aligned}
& u_{c, \varepsilon}(z)=-u_{c, \varepsilon}(-z), \\
& u_{c, \varepsilon}(z)=-u_{c, \varepsilon}(z+\mathrm{e}) .
\end{aligned}
$$


Note that (1.5) means that $u_{c, \varepsilon}$ is odd and periodic in the direction $\frac{\mathrm{e}}{|\mathrm{e}|}$ with minimal period $2 \mathrm{e}$. As we will see later on the nodal set of $u_{c, \varepsilon}$ is close to the family of curves $\left\{\left(t, q_{i}(t ; c, \varepsilon)\right)\right\}_{i \in \mathbb{Z}}$, where $\left\{q_{i}(t ; c, \varepsilon)\right\}_{i \in \mathbb{Z}}$ is the one-soliton solution of the Toda lattice given by (1.4). Observe that this set of curves is periodic in the direction $\frac{\mathrm{e}}{|\mathrm{e}|}$ with minimal period e. Figure 1 makes the situation transparent.
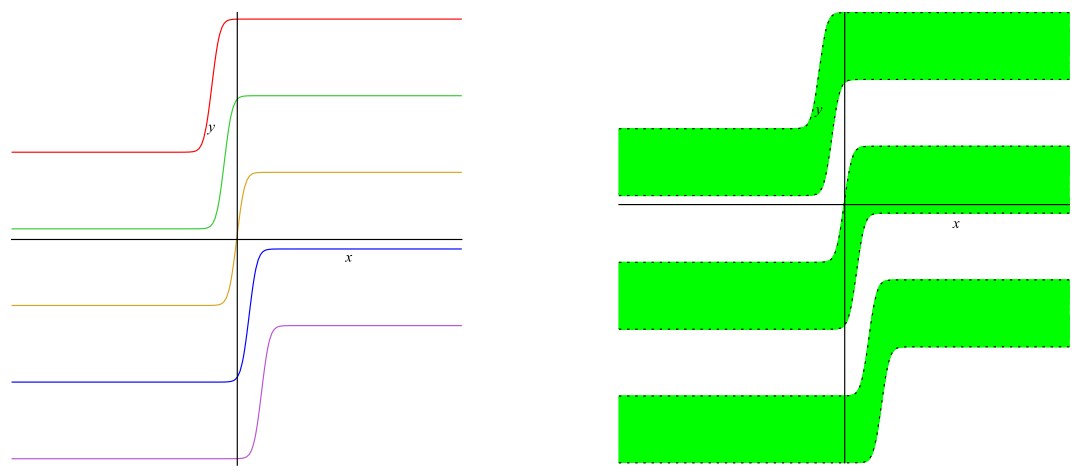

Figure 1. Plot of a scaled one-soliton solution to the Toda lattice (left) and a corresponding solution of the Allen-Cahn equation (right).

Let us finish this section by explaining the main ingredients of the proof. The main tool we will rely on is the infinite dimensional Lyapunov-Schmidt reduction, in a version which in this particular context has been implemented in [5]. Roughly speaking, the proof of the Theorem 1.1 will be split into three steps: first, a suitable family of approximate solutions, which is expected to be very close to the true solution, is constructed. Second, a perturbation term is added to these approximate solutions and a fixed point argument is applied to solve the Allen-Cahn equation, modulo the kernel of the linearized operator around the approximate solution. Finally, another fixed point argument is used to show that for some choices of the parameters involved in the approximate solutions, the projection onto the kernel is indeed equal to zero, which implies that the equation has been exactly solved and thus the desired solution of the original Allen-Cahn equation is obtained. This procedure depends in a crucial way on good understanding of the linear properties of the Toda lattice soliton and we devote the following section to this issue.

\section{The Toda Lattice AND its Linearization}

2.1. The linearized Toda lattice around the one-soliton. As we will see later, it will be important for us to analyze the linearized equation of the Toda lattice around the one-solition solution given by the functions $\left\{q_{i}\right\}_{i \in \mathbb{Z}}$. In particular, it will be crucial to understand the mapping property of the operator $\mathcal{L}$ :

$$
\varphi \longmapsto c_{*} \varphi^{\prime \prime}-\sqrt{2} e^{\sqrt{2}\left(q_{-1}(\cdot ; c, 1)-q_{0}(\cdot ; c, 1)\right)}\left(\varphi\left(\cdot+\alpha^{-1}\right)-\varphi\right)+\sqrt{2} e^{\sqrt{2}\left(q_{0}(\cdot ; c, 1)-q_{1}(\cdot ; c, 1)\right)}\left(\varphi-\varphi\left(\cdot-\alpha^{-1}\right)\right) .
$$

in the class of odd functions. Above and throughout the paper $\alpha=c \sqrt{\sqrt{2} c_{*}^{-1}}$.

Observe that after scaling of the operator $\mathcal{L}$ we obtain the operator $\mathcal{L}_{1}$ :

$$
\varphi \longmapsto \varphi^{\prime \prime}-e^{s_{-1}(\cdot ; c, 1)-s_{0}(\cdot ; c, 1)}\left(\varphi\left(\cdot+c^{-1}\right)-\varphi\right)+e^{s_{0}(\cdot ; c, 1)-s_{1}(\cdot ; c, 1)}\left(\varphi-\varphi\left(\cdot-c^{-1}\right)\right) .
$$

Therefore, for notational reasons, we will analyze the operator $\mathcal{L}_{1}$ instead of $\mathcal{L}$.

To begin with, let us introduce the weighted function spaces we will work with. Let $L_{a}^{2}(\mathbb{R}):=e^{a x} L^{2}(\mathbb{R})$ and $W_{a}^{2,2}(\mathbb{R}):=e^{a x} W^{2,2}(\mathbb{R})$. At the same time we will consider spaces $L_{a}^{2}\left(\mathbb{R}_{o}\right):=e^{a x} L^{2}\left(\mathbb{R}_{o}\right)$ and $W_{a}^{2,2}\left(\mathbb{R}_{o}\right):=$ $e^{a x} W^{2,2}\left(\mathbb{R}_{o}\right)$. Here the subscript $o$ of $\mathbb{R}$ indicates that the function spaces $L^{2}\left(\mathbb{R}_{o}\right)$ and $W^{2,2}\left(\mathbb{R}_{o}\right)$ involve only odd functions. Functions in the weighted spaces $L_{a}^{2}\left(\mathbb{R}_{o}\right)$ and $W_{a}^{2,2}\left(\mathbb{R}_{o}\right)$ do not have to be odd, in general. 
Initially, the introductions of spaces involving odd functions only does not seem to be justified, however later on the role of oddness will become apparent. Furthermore, let $\bar{L}_{a}^{2}(\mathbb{R})=(\cosh x)^{a} L^{2}(\mathbb{R})$ with the norm

$$
\|h\|_{\bar{L}_{a}^{2}(\mathbb{R})}=\left\|(\cosh x)^{-a} h\right\|_{L^{2}(\mathbb{R})} .
$$

Similarly, we define the space $\bar{W}_{a}^{2,2}(\mathbb{R})=(\cosh x)^{a} W^{2,2}(\mathbb{R})$, and

$$
\overline{\mathcal{C}}_{a}^{l, \mu}(\mathbb{R})=(\cosh x)^{a} \mathcal{C}^{l, \mu}(\mathbb{R})
$$

In what follows the Fourier transform $\hat{f}$ of $f$ and its inverse will be defined respectively by

$$
\hat{f}(\xi)=\int_{\mathbb{R}} f(x) e^{-2 \pi i \xi x} d x, \quad f(x)=\int_{\mathbb{R}} \hat{f}(\xi) e^{2 \pi i \xi x} d \xi .
$$

The following non degeneracy result for $\mathcal{L}_{1}$ will be used later.

Proposition 2.1. Let $a \in(-2 k, 2 k)$. Assume $\varphi \in W_{a}^{2,2}(\mathbb{R})$ and $\mathcal{L}_{1} \varphi=0$. Then necessary $\varphi=0$.

Essentially, up to some minor changes, this result was proved in [18] using a Bäcklund transformation. For completeness, we summarise the main steps of the proof, and refer to [18] for more details.

Lemma 2.1 (Lemma 3.1 of [7]). The zeros of the function

$$
p(\xi):=\xi^{2}-4 \sin ^{2}\left(\frac{\xi}{2 c}\right)=0,
$$

are symmetric across the real and imaginary lines. The set of zeros in the closure of the first quadrant is

$$
\{0,2 k i\} \cup\left\{a_{j}(c)+i b_{j}(c)\right\}_{j=1}^{+\infty},
$$

with

and $b_{j}>\frac{9}{2}$, for $j \geq 1$.

$$
2 j \pi<a_{j}(c)<2(j+1) \pi
$$

We introduce the operator $\mathcal{L}_{0}$ :

$$
\varphi \longmapsto \varphi^{\prime \prime}-\varphi\left(\cdot+c^{-1}\right)+\varphi\left(\cdot-c^{-1}\right)+2 \varphi
$$

Using Lemma 2.1, one can show the following

Lemma 2.2. Given $a \in \mathbb{R}$. Suppose $\varphi \in W_{a}^{2,2}(\mathbb{R})$ satisfies $\mathcal{L}_{0} \varphi=0$, then $\varphi \equiv 0$.

Proof. Let $\varphi=e^{a x} \eta$ with $\eta \in L^{2}(\mathbb{R})$. Then $\eta$ solves

$$
\eta^{\prime \prime}+2 a \eta^{\prime}+a^{2} \eta-e^{a c^{-1}} \eta\left(\cdot+c^{-1}\right)-e^{-a c^{-1}} \eta\left(\cdot-c^{-1}\right)+2 \eta=0 .
$$

Taking Fourier transform on both sides of (2.1), one gets

$$
p(i a+2 \pi \xi) \hat{\eta}(\xi)=0 .
$$

Using the result of Lemma 2.1, and the fact that $\eta \in L^{2}(\mathbb{R})$, we get $\eta=0$. This finishes the proof.

Proof of Proposition 2.1. The idea of the proof is to establish a correpondence between the kernel of $\mathcal{L}_{1}$ and $\mathcal{L}_{0}$, and use Lemma 2.2. We refer to [18] for details.

Now, we will study the mapping properties of the operator $\mathcal{L}_{0}$ on the weighted Sobolev spaces.

Lemma 2.3. The operator $\mathcal{L}_{0}$ is an isomorphism from $W_{a}^{2,2}(\mathbb{R})$ to $L_{a}^{2}(\mathbb{R})$ provided that $\mid$ a $\mid$ is small enough and $a \neq 0$.

Proof. By the above lemma, the operator is injective. Hence it suffices to solve the equation

$$
\mathcal{L}_{0} \varphi=f
$$

for each given $f \in L_{a}^{2}(\mathbb{R})$.

Write $f=e^{a x} F$ and $\varphi=e^{a x} \Phi$, with $F \in L^{2}(\mathbb{R})$ and $\Phi \in W^{2,2}(\mathbb{R})$. Taking Fourier transforms we find

$$
p(i a+2 \pi \xi) \hat{\Phi}=-\hat{F} \text {. }
$$


By the result of Lemma 2.1, for any $\xi \in \mathbb{R}, p(i a+2 \pi \xi) \neq 0$, if we suppose that $|a|$ is small and $a \neq 0$. Hence we get

$$
\|\hat{\Phi}\|_{L^{2}(\mathbb{R})} \leq C_{a}\|\hat{F}\|_{L^{2}(\mathbb{R})}
$$

where $C_{a}$ is a constant depending on $a$. Applying Plancherel's formula we complete the proof.

Since $\mathcal{L}_{1}$ is asymptotic to $\mathcal{L}_{0}$ at infinity, the above lemma can be used to prove the following:

Lemma 2.4. The operator $\mathcal{L}_{1}: \bar{W}_{a}^{2,2}(\mathbb{R}) \rightarrow \bar{L}_{a}^{2}(\mathbb{R})$ is Fredholm if $|a|$ is small and $a \neq 0$.

Proof. We first show the following estimate:

$$
\|u\|_{\bar{W}_{a}^{2,2}(\mathbb{R})} \leq C\left\|\mathcal{L}_{1} u\right\|_{\bar{L}_{a}^{2}(\mathbb{R})}+C\|u\|_{L^{2}\left(\left[-t_{0}, t_{0}\right]\right)} .
$$

where $t_{0}$ is a large constant to be determined later on. To do this, we let $\chi$ be a cutoff function equal to 1 in $\left[t_{0},+\infty\right)$ and equal to 0 in $\left(-\infty, t_{0}-1\right]$. By the results of Lemma 2.3,

$$
\|\chi u\|_{L_{a}^{2}(\mathbb{R})} \leq C\left\|\mathcal{L}_{0}(\chi u)\right\|_{L_{a}^{2}(\mathbb{R})} .
$$

On the other hand, using asymptotic behaviour of the one-soliton, we find that if $t_{0}$ is large enough, then

$$
\left\|\mathcal{L}_{0}(\chi u)\right\|_{L_{a}^{2}(\mathbb{R})} \leq C\left\|\mathcal{L}_{1}(\chi u)\right\|_{L_{a}^{2}(\mathbb{R})}+\delta\|\chi u\|_{L_{a}^{2}(\mathbb{R})},
$$

for some small $\delta>0$. Similar estimate can be proven for $L_{-a}^{2}(\mathbb{R})$ norms, respectively of $\chi(-t) u, \mathcal{L}_{0}(\chi(-t) u)$ and $\mathcal{L}_{1}(\chi(-t) u)$. This together with (2.3) readily implies $(2.2)$. By elliptic estimates we get

$$
\|u\|_{\bar{W}_{a}^{2,2}(\mathbb{R})} \leq C\left\|\mathcal{L}_{1} u\right\|_{\bar{L}_{a}^{2}(\mathbb{R})}+C\|u\|_{L^{2}\left(\left[-t_{0}, t_{0}\right]\right)} .
$$

from which the Fredholm property follows by a standard argument (see for instance [19] or [4]) .

Another property of $\mathcal{L}_{1}$ we shall use later on is contained in the following

Proposition 2.2 (Linear decomposition lemma). Let $a>0$ be small. Suppose $u \in \bar{W}_{a}^{2,2}(\mathbb{R})$ satisfies $\mathcal{L}_{1} u=f$ with $f \in \bar{L}_{-a}^{2}(\mathbb{R})$. Then there exists $\phi \in \bar{W}_{-a}^{2,2}(\mathbb{R})$ and constants $c_{1}^{ \pm}$, $c_{2}^{ \pm}$such that

$$
u(x)=\phi(x)+\left(c_{1}^{+}+c_{2}^{+} x\right) \chi_{0}^{+}(x)+\left(c_{1}^{-}+c_{2}^{-} x\right) \chi_{0}^{-}(x),
$$

where $\chi_{0}^{+}$is a smooth cut off function equal to 1 in $[0,+\infty)$ and equal to 0 in $(-\infty,-1]$, and $\chi_{0}^{-}$is a smooth cut off function such that $\chi_{0}^{+}+\chi_{0}^{-} \equiv 1$.

Proof. Recall that $\mathcal{L}_{1}-\mathcal{L}_{0}$ decays exponentially fast at infinity. Therefore if $a>0$ is small enough, then

$$
\mathcal{L}_{0} u=\mathcal{L}_{1} u+\mathcal{L}_{0} u-\mathcal{L}_{1} u:=\tilde{f} \in \bar{L}_{-a}^{2}(\mathbb{R}) .
$$

We also have $\chi_{0}^{+} u \in W_{a}^{2,2}(\mathbb{R})$ and

$$
\mathcal{L}_{0}\left(\chi_{0}^{+} u\right)=f_{1},
$$

where $f_{1} \in L_{-a}^{2}(\mathbb{R})$. We write $u=\chi_{0}^{+} u+\chi_{0}^{-} u$ and

$$
\chi_{0}^{+}(x) u(x)=e^{a x} w(x), \quad w \in W^{2,2}(\mathbb{R}) .
$$

It suffices to show that

$$
e^{a x} w-\left(c_{1}+c_{2} x\right) \chi_{0}^{+} \in L_{-a}^{2}(\mathbb{R}),
$$

for some constants $c_{1}$ and $c_{2}$ (the argument for $\chi_{0}^{-} u$ being similar).

Let $f_{2}=e^{-a x} f_{1} \in L_{-2 a}^{2}(\mathbb{R})$. Similarly as before, the Fourier transform $\hat{w}$ of $w$ satisfies

$$
\hat{w}=\frac{-\hat{f}_{2}}{p(i a+2 \pi \xi)} .
$$

Then

$$
w(x)=\int_{\mathbb{R}} \frac{-\hat{f}_{2}(\xi)}{p(i a+2 \pi \xi)} e^{2 \pi i \xi x} d \xi
$$


This implies

$$
\begin{aligned}
& w^{\prime \prime}+2 a w^{\prime}+a^{2} w \\
& =\int_{\mathbb{R}} \frac{(-i a+2 \pi \xi)^{2} \hat{f}_{2}(\xi)}{p(i a+2 \pi \xi)} e^{2 \pi i \xi x} d \xi:=g(x) .
\end{aligned}
$$

Let us compute

$$
\begin{aligned}
g(x) e^{2 a x} & =\int_{\mathbb{R}} \frac{(-i a+2 \pi \xi)^{2} \hat{f}_{2}(\xi)}{p(i a+2 \pi \xi)} e^{\left(\xi-\frac{i a}{\pi}\right) 2 \pi i x} d \xi \\
& =\int_{\mathbb{R}-\frac{a i}{\pi}} \frac{(i a+2 \pi \bar{\xi})^{2} \hat{f}_{2}\left(\bar{\xi}+\frac{i a}{\pi}\right)}{p(3 i a+2 \pi \bar{\xi})} e^{2 \pi i \bar{\xi} x} d \bar{\xi} .
\end{aligned}
$$

Recall that

$$
\begin{aligned}
\hat{f}_{2}\left(\bar{\xi}-\frac{a}{\pi i}\right) & =\int_{\mathbb{R}} e^{-a x} f_{1}(x) e^{-2 \pi i\left(\bar{\xi}-\frac{a}{\pi i}\right) x} d x \\
& =\int_{\mathbb{R}} e^{a x} f_{1}(x) e^{-2 \pi i \bar{\xi} x} d x .
\end{aligned}
$$

Therefore,

$$
g(x) e^{2 a x}=\int_{\mathbb{R}-\frac{a i}{\pi}} \frac{(i a+2 \pi \bar{\xi})^{2} \int_{\mathbb{R}} e^{a x} f_{1}(x) e^{-2 \pi i \bar{\xi} x} d x}{p(3 i a+2 \pi \bar{\xi})} e^{2 \pi i \bar{\xi} x} d \bar{\xi} .
$$

This together with $f_{1} \in \bar{L}_{-a}^{2}(\mathbb{R})$ and the property of $p$ implies that $g \in L_{-2 a}^{2}(\mathbb{R})$. From

$$
w^{\prime \prime}+2 a w^{\prime}+a^{2} w=g,
$$

we infer that $e^{a x} w-\left(c_{1}+c_{2} x\right) \chi_{0}^{+} \in L_{-a}^{2}(\mathbb{R})$ for some constants $c_{1}$ and $c_{2}$.

Using similar arguments, one gets an analogous result in weighted Hölder spaces.

Lemma 2.5. Assume $a>0$ is small enough. Suppose $\mathcal{L}_{1} u=f$ with $f \in \overline{\mathcal{C}}_{-a}^{0, \mu}(\mathbb{R})$ and $u \in L^{\infty}(\mathbb{R})$, then there exists $\phi \in \overline{\mathcal{C}}_{-a}^{2, \mu}(\mathbb{R})$ and constants $c^{+}, c^{-}$, such that

$$
u(x)=\phi(x)+\chi_{0}(x) c^{+}+\chi_{0}(x) c^{-} .
$$

Proof. If $a>0$ is small enough, then

$$
\mathcal{L}_{0} u=f_{1} \in \overline{\mathcal{C}}_{-a}^{0, \mu}(\mathbb{R}) .
$$

Taking Fourier transform $\left(u \in L^{\infty}(\mathbb{R})\right.$ is a distribution), one gets

$$
\hat{u}(\xi)=\frac{-\hat{f}_{1}(\xi)}{p(2 \pi \xi)}
$$

and

Hence

$$
u(x)=\int_{\mathbb{R}} \frac{-\hat{f}_{1}(\xi)}{p(2 \pi \xi)} e^{2 \pi i \xi x} d \xi
$$

$$
u^{\prime \prime}(x)=\int_{\mathbb{R}} \frac{-(2 \pi \xi)^{2} \hat{f}_{1}(\xi)}{p(2 \pi \xi)} e^{2 \pi i \xi x} d \xi:=g(x) .
$$

Denote $e^{a x} f_{1}=f_{2} \in \overline{\mathcal{C}}^{0, \mu}(\mathbb{R})$. Substituting in the above expression we get

$$
g(x) e^{a x}=-\int_{\mathbb{R}} f_{2}(y)\left[e^{a(x-y)} \int_{\mathbb{R}} \frac{(2 \pi \xi)^{2}}{p(2 \pi \xi)} e^{2 \pi i \xi(x-y)} d \xi\right] d y=-f_{2} * h,
$$

where

$$
h(x)=e^{a x} \tilde{p}(x), \quad \tilde{p}(x)=\mathcal{F}^{-1}\left[\frac{(2 \pi \xi)^{2}}{p(2 \pi \xi)}\right](x)
$$


and $\mathcal{F}^{-1}$ is the inverse Fourier transform. It can be shown that $\tilde{p}$ is positive (Lemma 5.1 of [7]) and exponentially decaying at infinity at the rate $\cosh ^{-2 k(c)}(x)$, where $2 k(c)>0$ is the zero of $p$ which is the closets to the real axis (see the poof of Lemma 5.4 of [7]). Consequently, when $|a|$ is small enough

$$
u^{\prime \prime} \in e^{-a x} L^{\infty}(\mathbb{R}) .
$$

This, together with $u \in L^{\infty}(\mathbb{R})$ readily implies the lemma.

The above results lead to the definition of the deficiency space

$$
\mathcal{D}:=\operatorname{Span}\left\{\chi_{0}^{ \pm}, x \chi_{0}^{ \pm}\right\}
$$

We want to study the mapping properties of a densely defined operator

$$
\begin{aligned}
\mathcal{T}_{a}: \bar{L}_{a}^{2}(\mathbb{R}) & \longrightarrow \bar{L}_{a}^{2}(\mathbb{R}), \\
u & \longmapsto \mathcal{L}_{1} u .
\end{aligned}
$$

By the above results $\mathcal{T}_{a}$ is Fredholm (Lemma 2.4). If $|a|$ is small and $a>0$ then $\mathcal{T}_{-a}$ is injective and then by duality $\mathcal{T}_{a}$ is surjective. Additionally we define a densely defined operator:

$$
\begin{aligned}
\mathrm{T}_{a}: \bar{L}_{a}^{2}(\mathbb{R}) \oplus \mathcal{D} & \longrightarrow \bar{L}_{a}^{2}(\mathbb{R}), \\
u & \longmapsto \mathcal{L}_{1} u .
\end{aligned}
$$

The Linear Decomposition Lemma says that $\mathrm{T}_{-a}$ is surjective when $a>0$ is small and so in order to define the inverse of $\mathcal{L}_{1}$ (or in other words determine the "smallest" space in which $\mathcal{L}_{1}$ is an isomorphism) it remains to find the kernel of $\mathcal{L}_{1}$.

Lemma 2.6. Let $a>0$ be small. The dimension of the kernel of $\mathrm{T}_{-a}$ is 2 and there exists a two dimensional linear subspace $\mathcal{E}$ such that $\mathcal{D}=\operatorname{Ker}_{-a} \oplus \mathcal{E}$ and the operator $\mathcal{L}_{1}: \bar{W}_{-a}^{2,2}(\mathbb{R}) \oplus \mathcal{E} \rightarrow \bar{L}_{-a}^{2}(\mathbb{R})$ is an isomorphism.

Proof. The proof of this Lemma, based on the Relative Index Formula, is quite standard (see [17], [19], [4] among others). The argument goes as follows: by the Linear Decomposition Lemma

$$
\operatorname{Ker} \mathcal{T}_{a}=\operatorname{Ker}_{-a} .
$$

By duality

$$
\operatorname{dim} \operatorname{Ker} \mathcal{T}_{a}=\operatorname{codim} \operatorname{Im} \mathcal{T}_{-a} .
$$

By the Linear Decomposition Lemma we also have

$$
\operatorname{dim} \mathcal{D}=\operatorname{dim} \operatorname{Ker} \mathcal{T}_{a}+\operatorname{codim} \operatorname{Im} \mathcal{T}_{-a} .
$$

Then we find

$$
\operatorname{dim} \operatorname{Ker}_{-a}=\frac{1}{2} \operatorname{dim} \mathcal{D}=2,
$$

from which the assertion of the Lemma follows immediately.

Now, we turn to considering functions which are odd. We define spaces $\bar{L}_{a}^{2}\left(\mathbb{R}_{o}\right), \bar{W}_{a}^{2,2}\left(\mathbb{R}_{o}\right)$ and $\overline{\mathcal{C}}_{a}^{l, \mu}\left(\mathbb{R}_{o}\right)$ to be, respectively, the subspaces of $\bar{L}_{a}^{2}(\mathbb{R}), \bar{W}_{a}^{2,2}(\mathbb{R})$ and $\overline{\mathcal{C}}_{a}^{l, \mu}(\mathbb{R})$ which consist of odd functions. We introduce a two dimensional deficiency space

$$
\mathcal{D}_{o}=\operatorname{Span}\left\{\chi_{0}^{+}-\chi_{0}^{-}, x\right\}
$$

Without loss of generality we may assume that $\chi_{0}^{+}(x)=\chi_{0}^{-}(-x)$ and thus $\mathcal{D}_{o}$ consists of odd functions. We also define a one dimensional subspace

$$
\mathcal{E}_{o}=\operatorname{Span}\left\{\chi_{0}^{+}-\chi_{0}^{-}\right\}
$$

which consist of odd functions which are asymptotically constant at infinity.

With all these preparation, we state the following result:

Proposition 2.3. Let $a>0$ with $|a|$ small. Then the operator $\mathcal{L}_{1}$ is an isomorphism from $\bar{W}_{-a}^{2,2}\left(\mathbb{R}_{o}\right) \oplus \mathcal{E}_{o}$ to $\bar{L}_{-a}^{2}\left(\mathbb{R}_{o}\right)$. 
Proof. By the results of Lemma 2.6 to prove the proposition it suffices to find an element of the kernel of $\mathcal{L}_{1}$ which is odd and linear at infinity. Let us construct it explicitly.

Recall that the functions $s_{i}(t ; c, \lambda)=S_{c}(i-c \lambda t)-2 i \ln \lambda, \lambda>0$ determine a two parameter family of solutions to the Toda lattice equation:

$$
s_{i}^{\prime \prime}=e^{s_{i-1}-s_{i}}-e^{s_{i}-s_{i+1}} .
$$

Therefore, $\left\{\frac{\partial}{\partial c} s_{i}(\cdot, c, 1)\right\}_{i=1}^{n}$ and $\left\{\left.\frac{\partial}{\partial \lambda} s_{i}(\cdot, c, \lambda)\right|_{\lambda=1}\right\}_{i=1}^{n}$ will be solutions of the linearized Toda lattice equation:

$$
\xi_{i}^{\prime \prime}(t)=e^{s_{i-1}(\cdot ; c, 1)-s_{i}(\cdot ; c, 1)}\left(\xi_{i-1}-\xi_{i}\right)-e^{s_{i}(\cdot ; c, 1)-s_{i+1}(\cdot ; c, 1)}\left(\xi_{i}-\xi_{i+1}\right), \quad i \in \mathbb{Z} .
$$

Obviously, for any constant $v$, the function

$$
t \rightarrow s_{i}(t ; c, \lambda)+v t, \quad i \in \mathbb{Z},
$$

will also be a solution of the Toda equation. Differentiating this function with respect to $v$, we find another solution of (2.6) which is the linear, odd function $t$. We have

$$
\begin{aligned}
& \left.\frac{\partial s_{i}}{\partial c}\right|_{\lambda=1}=\left.\partial_{c} S_{c}(x)\right|_{\lambda=1, x=i-c t}-\varepsilon t S_{c}^{\prime}(i-c t):=\Xi_{i}, \\
& \left.\frac{\partial s_{i}}{\partial \lambda}\right|_{\lambda=1}=-c t S_{c}^{\prime}(i-c t)-2 i:=\Phi_{i} .
\end{aligned}
$$

We claim that there exist constants $k_{1}, k_{2}, k_{3}$ such that the function

$$
K_{i}(t):=k_{1} \Xi_{i}+k_{2} \Phi_{i}+k_{3} t
$$

is unbounded, odd and satisfies $K_{i}\left(t+c^{-1}\right)=K_{i-1}(t)$. Indeed, the above condition reads

$$
k_{1}\left(\Xi_{i}\left(t+c^{-1}\right)-\Xi_{i}(t)\right)+k_{2}\left(\Phi_{i}\left(t+c^{-1}\right)-\Phi_{i}(t)\right)+k_{3} c^{-1}=0 .
$$

We calculate directly

$$
\begin{gathered}
\Xi_{i}\left(t+c^{-1}\right)-\Xi_{i}(t)=-c^{-1} S_{c}^{\prime}(i-1-c t), \\
\Phi_{i}\left(t+c^{-1}\right)-\Phi_{i}(t)=S_{c}^{\prime}(i-1-c t)+2,
\end{gathered}
$$

hence it suffices to choose $k_{1}=1, k_{2}=c^{-1}, k_{3}=-2$.

Accepting this claim for now (its proof is given in the appendix) we see that $K_{0}$ is the desired element of the kernel of $\mathcal{L}_{1}$.

Actually, we have similar result in weighted Hölder spaces:

Proposition 2.4. Let $a>0$ with $|a|$ small. The operator $\mathcal{L}_{1}: \bar{C}_{-a}^{2, \mu}\left(\mathbb{R}_{o}\right) \oplus \mathcal{E}_{o} \rightarrow \bar{C}_{-a}^{0, \mu}\left(\mathbb{R}_{o}\right)$ is an isomorphism.

Proof. By the results of Proposition 2.3, $\mathcal{L}_{1}$ is injective. Hence it is enough to show that it is also surjective. Given $f \in \overline{\mathcal{C}}_{-a}^{0, \mu}\left(\mathbb{R}_{o}\right)$, let us show the existence of $\phi \in \overline{\mathcal{C}}_{-a}^{2, \mu}\left(\mathbb{R}_{o}\right) \oplus \mathcal{E}_{o}$, a solution of $\mathcal{L}_{1} \phi=f$.

Certainly, $f \in \bar{L}_{-a^{\prime}}^{2}\left(\mathbb{R}_{o}\right)$, for $a^{\prime} \in(0, a)$. By Proposition 2.3, we can find $\phi \in \bar{W}_{a^{\prime}}^{2,2}\left(\mathbb{R}_{o}\right) \oplus \mathcal{E}_{o}$ such that $\mathcal{L}_{1} \phi=f$. By elliptic regularity, $\phi \in L^{\infty}(\mathbb{R})$. Since $f \in \overline{\mathcal{C}}_{-a}^{0, \mu}\left(\mathbb{R}_{o}\right)$, by Lemma $2.5, \phi \in \overline{\mathcal{C}}_{-a}^{2, \mu}\left(\mathbb{R}_{o}\right) \oplus \mathcal{E}_{o}$. This finishes the proof.

\section{A family of approximate solutions}

We recall that $\alpha=c \sqrt{\sqrt{2} c_{*}^{-1}}$ and $c_{*}=\frac{\sqrt{2}}{24}$ is the constant appearing in (1.3). Let $\mathrm{e}=\mathrm{e}_{c, \varepsilon}:=\left(\frac{1}{\alpha \varepsilon}, \sqrt{2}|\ln \varepsilon|\right)$. Note that the set of curves $\left\{\left(x, q_{i}(x ; c, \varepsilon)\right)\right\}_{i \in \mathbb{Z}}$ in $\mathbb{R}^{2}$ is periodic in the direction $\frac{\mathrm{e}}{|\mathrm{e}|}$ with minimal period $|\mathrm{e}|$ (we omit the subscript in $\mathrm{e}_{c, \varepsilon}$ for simplification). For each $\varepsilon>0$ small enough, we will construct a solution $u_{\varepsilon}$ of the Allen-Cahn equation which is odd and periodic in the direction $\frac{\mathrm{e}}{|\mathrm{e}|}$ with minimal period $2|\mathrm{e}|$. These two conditions are equivalent to

$$
u(z)=-u(-z), \quad u(z)=-u(z+\mathrm{e}) .
$$

An important step towards this aim will be the construction of suitable approximate solutions. As we will see, the nodal sets of these functions will be very close to the graphs of the one-soliton solution to the Toda lattice discussed in the last section. 
We first describe the Fermi coordinates $\left(x_{i}, y_{i}\right), i \in \mathbb{Z}$, associated to the curve $\Gamma_{i}$, which is the graph of the function $y=q_{i}(x)$ (for brevity we will write $q_{i}(x)$ for $q_{i}(x ; c, \varepsilon)$ from now on). For a tubular neighbourhood $\mathfrak{T}_{i}$ (as we will see later, its radius will be $\mathcal{O}\left(\varepsilon^{-1}\right)$ ) of $\Gamma_{i}$, consider the map $X_{i}$ given by

$$
\left(x_{i}, y_{i}\right) \longmapsto(x, y)=\left(x_{i}, q_{i}\left(x_{i}\right)\right)+y_{i} n_{i}\left(x_{i}\right) \in \mathfrak{T}_{i},
$$

where $n_{i}$ is the unit normal vector of the curve $\Gamma_{i}$ at the point $\left(x_{i}, q_{i}\left(x_{i}\right)\right)$ :

$$
n_{i}\left(x_{i}\right)=\frac{\left(-q_{i}^{\prime}\left(x_{i}\right), 1\right)}{\sqrt{1+\left(q_{i}^{\prime}\left(x_{i}\right)\right)^{2}}},
$$

and $y_{i}$ is the signed distance from the point $(x, y)$ to the curve $\Gamma_{i}$, positive in the upper part of $\mathbb{R}^{2} \backslash \Gamma_{i}$ and negative in the lower part. This map $X_{i}$ gives a parametrization of $\mathfrak{T}_{i}$. Since the slope of the graph of $q_{i}$ is of order $\mathcal{O}(\varepsilon)$ and $\left(x, q_{i}(x)\right)$ is asymptotically horizontal, without difficulty one can show the following.

Lemma 3.1. There exists a constant $C>0$ such that the map $X_{i}$ is a diffeomorphism from $\mathbb{R} \times\left(-C \varepsilon^{-1}, C \varepsilon^{-1}\right)$ onto

$$
\mathfrak{T}_{i}:=\left\{z \in \mathbb{R}^{2} \mid \mathrm{d}\left(\Gamma_{i}, z\right)<C \varepsilon^{-1}\right\} .
$$

For each function $f: \mathfrak{T}_{i} \rightarrow \mathbb{R}$, the following convenient notation will be adopted:

$$
X_{i}^{*} f\left(x_{i}, y_{i}\right)=f \circ X_{i}\left(x_{i}, y_{i}\right) .
$$

Let $\beta_{0}>0$ be a constant to be fixed later. Given $\delta>0$ small, and $h \in C_{-a}^{2, \mu}\left(\mathbb{R}_{o}\right) \oplus \mathcal{E}_{o}$, with

$$
\|h\|_{C_{-\delta}^{2, \mu}\left(\mathbb{R}_{o}\right) \oplus \mathcal{E}_{o}} \leq \varepsilon^{\beta_{0}},
$$

let us denote

$$
h_{i}(x)=h\left(x-i \alpha^{-1}\right) .
$$

Next, functions $H_{i}=H_{i}(\cdot ; h)$ and $H_{i}^{\prime}=H_{i}^{\prime}(\cdot ; h)$, with $h$ being a parameter, are defined through the relation

$$
\begin{aligned}
& X_{i}^{*} H_{i}\left(x_{i}, y_{i} ; h\right)=H\left(y_{i}-h_{i}\left(\varepsilon x_{i}\right)\right), \\
& X_{i}^{*} H_{i}^{\prime}\left(x_{i}, y_{i} ; h\right)=H^{\prime}\left(y_{i}-h_{i}\left(\varepsilon x_{i}\right)\right) .
\end{aligned}
$$

We recall that $H$ is the unique, odd, monotonically increasing solution of $-H^{\prime \prime}=H-H^{3}$. Choose a smooth nonnegative function $\rho$ satisfying

$$
\rho(s)= \begin{cases}1, & s<-1 \\ 0, & s>1\end{cases}
$$

and $\rho(s)+\rho(-s)=1$. Fix a small number $\varsigma$ and define $\rho_{\varsigma}(s)=\rho(\varsigma s)$ Denote

$$
\gamma_{i}(x):=\frac{q_{i}(x)-q_{i-1}(x)}{2}, \quad i \in \mathbb{Z}
$$

We define a cutoff function

$$
\eta_{0}(x, y):= \begin{cases}\rho_{\varsigma}\left(y-q_{0}(x)-\gamma_{1}(x)\right), & y>q_{0}(x) \\ \rho_{\varsigma}\left(-y+q_{0}(x)-\gamma_{0}(x)\right), & y \leq q_{0}(x) .\end{cases}
$$

Since $q_{0}$ is an odd function, one may check that $\eta_{0}(z)=\eta_{0}(-z)$. We set

$$
\eta_{i}(z)=\eta_{0}(z-i \mathrm{e}), i \in \mathbb{Z}
$$

$\eta_{i}$ is supported in the region

$$
\Omega_{i}:=\left\{z \in \mathbb{R}^{2}: z=\left(x, q_{i}(x)+t\right), t \in\left(-\gamma_{i}(x)-\frac{1}{\varsigma}, \gamma_{i+1}(x)+\frac{1}{\varsigma}\right)\right\} .
$$

By construction,

$$
\sum_{i=-\infty}^{+\infty} \eta_{i}(z)=1
$$


Although, for brevity, we do not indicate the dependence of the functions $\eta_{i}$ on $\varsigma$ we keep in mind that eventually $\varsigma$ needs to be adjusted. This is because we will need

$$
\left|\nabla \eta_{i}\right|+\left|D^{2} \eta_{i}\right| \leq C \varsigma=o(1), \quad \varepsilon \rightarrow 0
$$

which certainly can be achieved since $\gamma_{i}=\mathcal{O}(|\ln \varepsilon|)$.

Note that $\eta_{i}$ actually depends on the parameters $c$ and $\varepsilon$, although we have not expressed this explicitly. For future purpose, we need another family of cutoff functions $\hat{\eta}_{i}, i \in \mathbb{Z}$, given by

Note the relation

$$
\hat{\eta}_{0}(x, y)=\eta_{0}\left(x, \frac{2}{5} y\right), \quad \hat{\eta}_{i}(z)=\hat{\eta}_{0}(z-i \mathrm{e}) .
$$

$$
\eta_{i} \hat{\eta}_{i}=\eta_{i}
$$

The curve $\Gamma_{i}$ is not a straight line, hence the Fermi coordinate $\left(x_{i}, y_{i}\right)$ is not well defined in the whole plane. A direct consequence is that the functions $H_{j}$ are also not defined on the whole space. To overcome this difficulty, we take advantage of the cutoff functions $\hat{\eta}_{i}$ to define

$$
\mathcal{H}_{j}(\cdot ; h):=\hat{\eta}_{j} H_{j}(\cdot ; h)+\left(1-\hat{\eta}_{j}\right) \frac{H_{j}}{\left|H_{j}\right|}, i \in \mathbb{Z} .
$$

With all these notations at hand, we shall define an approximate solution $\mathfrak{u}$ to the Allen-Cahn equation through a formal series

$$
\mathfrak{u}=\sum_{i=1}^{\infty}(-1)^{i} \mathcal{H}_{i}
$$

With this definition infinitely many terms of the series are equal 1 or -1 and we agree that they cancel each other, so that for any $z \in \Omega_{i}$,

$$
\mathfrak{u}(z):=(-1)^{i}\left(-\mathcal{H}_{i-1}(z)+\mathcal{H}_{i}(z)-\mathcal{H}_{i+1}(z)\right) .
$$

Using the definition of $\mathcal{H}_{i}$, one verifies directly that $\mathfrak{u}$ is a smooth function. Using the symmetry and periodicity of the family of curves $\left\{\Gamma_{i}\right\}_{i \in \mathbb{Z}}$ we find

$$
\mathfrak{u}(z)=-\mathfrak{u}(z+\mathrm{e}), \quad \mathfrak{u}(-z)=-\mathfrak{u}(z) .
$$

Note that the approximate solution depends on the still undetermined function $h$, we will indicate it by writing $\mathfrak{u}(z ; h)$ whenever necessary.

\section{From approximate solutions to Genuine solutions}

Recall that in the Fermi coordinate $\left(x_{i}, y_{i}\right)$, the expression for the Laplacian operator is given by (see Section 5.1 of [5]):

$$
\Delta=\frac{1}{A_{i}} \partial_{x_{i}}^{2}+\partial_{y_{i}}^{2}+\frac{1}{2} \frac{\partial_{y_{i}} A_{i}}{A_{i}} \partial_{y_{i}}-\frac{1}{2} \frac{\partial_{x_{i}} A_{i}}{A_{i}^{2}} \partial_{x_{i}}
$$

where

$$
A_{i}=1+\left(q_{i}^{\prime}\left(x_{i}\right)\right)^{2}-2 y_{i} \frac{q_{i}^{\prime \prime}\left(x_{i}\right)}{\sqrt{1+\left(q_{i}^{\prime}\left(x_{i}\right)\right)^{2}}}+y_{i}^{2} \frac{\left(q_{i}^{\prime \prime}\left(x_{i}\right)\right)^{2}}{\left(1+\left(q_{i}^{\prime}\left(x_{i}\right)\right)^{2}\right)^{2}}
$$

In the region $\Omega_{i}$, by definition

$$
(-1)^{i} \mathfrak{u}=-\mathcal{H}_{i-1}+\mathcal{H}_{i}-\mathcal{H}_{i+1}
$$

If we denote by

$$
E(\mathfrak{u}):=\Delta \mathfrak{u}+\mathfrak{u}-\mathfrak{u}^{3}
$$

the error of the approximate solution, then a first glimpse at (4.1) tells us that $E(\mathfrak{u})$ does not decay exponentially fast as $|x| \rightarrow+\infty$. Indeed, the heteroclinic solution $H(y)=\tanh \left(\frac{y}{\sqrt{2}}\right)$ and thus $H(y)-1$, (respectively $H(y)+1)$ and $H^{\prime}(y)$ decay exponentially as $|y| \rightarrow \infty$. This means that the error of the approximation is globally small, of order $\mathcal{O}\left(e^{-c / \varepsilon}\right)$. However, due to the fact that the level set of $\mathfrak{u}$ consists of curves whose mutual distances at infinity are constant there is no hope for an estimate of the error in a norm that takes 
into account decay both in the direction orthogonal and parallel to the level set. This makes our present situation different than that of the multiple end solutions considered in [4] or [16]. As we will see later on in order to deal with this difficulty we take advantage of the fact that at infinity our equation reduces essentially to a one dimensional periodic problem.

4.1. The infinite dimensional reduction. We want to find a suitable function $h$ and a corresponding small perturbation $\phi=\phi(\cdot ; h)$ of $\mathfrak{u}$, such that $\mathfrak{u}+\phi$ is a genuine solution of the Allen-Cahn equation. If such a $\phi$ exists, then it should satisfy

$$
\mathcal{T} \phi=E(\mathfrak{u})-P(\mathfrak{u}, \phi),
$$

where

$$
\mathcal{T} \phi=-\Delta \phi+\left(3 \mathfrak{u}^{2}-1\right) \phi, \quad P(\mathfrak{u}, \phi)=3 \mathfrak{u} \phi^{2}+\phi^{3} .
$$

To get a solution to equation (4.2), we suppose that $\phi$ can be written as

$$
\phi=\sum_{i \in \mathbb{Z}} \eta_{i} \phi_{i}^{\#} .
$$

The unknown functions $\phi_{i}^{\#}$ are not globally defined in the whole $\mathbb{R}^{2}$ but only in some open neighbourhood on the sets $\operatorname{supp} \eta_{i}$. In reality, because our problem is periodic we only need to determine the function $\phi_{0}$ in such a way that

$$
\phi_{0}^{\#}(z)=-\phi_{0}^{\#}(-z) .
$$

Then we set

$$
\phi_{i}^{\#}(z)=(-1)^{i} \phi_{0}^{\#}(z-i \mathrm{e}) .
$$

It is elementary to verify that with this definition we have (recall that $\eta_{i}(z)=\eta_{0}(z-i \mathbf{e})$ and $\eta_{0}$ is odd)

$$
\phi(z)=-\phi(-z) \quad \text { and } \quad \phi(z)=-\phi(z+\mathrm{e}) .
$$

The equation under consideration is

$$
\sum_{i \in \mathbb{Z}} \mathcal{T}\left(\eta_{i} \phi_{i}^{\#}\right)=E(\mathfrak{u})-P(\mathfrak{u}, \phi) .
$$

For each $i \in \mathbb{Z}$ we introduce the operator

$$
L_{i} \varphi:=-\Delta \varphi+\left(3 H_{i}^{2}-1\right) \varphi .
$$

The equation (4.4) can be rewritten as

$$
\sum_{i \in \mathbb{Z}}\left(L_{i}\left(\eta_{i} \phi_{i}^{\#}\right)+\left(\mathcal{T}-L_{i}\right)\left(\eta_{i} \phi_{i}^{\#}\right)\right)=E(\mathfrak{u})-P(\mathfrak{u}, \phi) .
$$

This is equivalent to

$$
\begin{aligned}
\sum_{i \in \mathbb{Z}}\left(\eta_{i} L_{i} \phi_{i}^{\#}\right) & =E(\mathfrak{u})-P(\mathfrak{u}, \phi) \\
& -\sum_{i \in \mathbb{Z}}\left(\mathcal{T}-L_{i}\right)\left(\eta_{i} \phi_{i}^{\#}\right) \\
& -\sum_{i \in \mathbb{Z}}\left[L_{i}, \eta_{i}\right] \phi_{i}^{\#} .
\end{aligned}
$$

Here

$$
\left[L_{i}, \eta_{i}\right]=L_{i} \circ \eta_{i}-\eta_{i} \circ L_{i}
$$


is the commutator of $L_{i}$ and $\eta_{i}$. Hence, to find a solution $\phi$ of equation (4.2), it will suffice to solve the following equation for the function $\phi_{0}^{\#}$ :

$$
\begin{aligned}
L_{0} \phi_{0}^{\#} & =\hat{\eta}_{0} E(\mathfrak{u})-\hat{\eta}_{0} P(\mathfrak{u}, \phi) \\
& -\hat{\eta}_{0} \sum_{i \in \mathbb{Z}}\left(\mathcal{T}-L_{i}\right)\left(\eta_{i} \phi_{i}^{\#}\right)-\hat{\eta}_{0} \sum_{i \in \mathbb{Z}}\left[L_{i}, \eta_{i}\right] \phi_{i}^{\#} \\
& :=\mathcal{F} .
\end{aligned}
$$

Indeed, multiplying (4.6) by $\eta_{0}$, using (3.4) and (4.3) we get (4.5).

If we denote $\phi_{i}=X_{i}^{*} \phi_{i}^{\#}$ and introduce the operator $L_{0}^{*}$ through

$$
L_{0}^{*} \zeta:=X_{0}^{*}\left(L_{0}\left(\zeta \circ X_{0}^{-1}\right)\right),
$$

then the equation satisfied by $\phi_{0}$ is

$$
L_{0}^{*} \phi_{0}=X_{0}^{*} \mathcal{F} .
$$

Note that since $\phi_{0}^{\#}$ is odd (4.3) and in the set $\mathfrak{T}_{0}$ we have

$$
z=X_{0}\left(x_{0}, y_{0}\right) \Longrightarrow-z=X_{0}\left(-x_{0},-y_{0}\right),
$$

therefore

$$
\phi_{0}\left(x_{0}, y_{0}\right)=\phi_{0}^{\#} \circ X_{0}\left(x_{0}, y_{0}\right)=-\phi_{0}^{\#} \circ X_{0}\left(-x_{0},-y_{0}\right)=-\phi\left(-x_{0},-y_{0}\right),
$$

which mean that $\phi_{0}$ should be odd. At first it seems that $\phi_{0}$ needs to be defined only in the set $\mathbb{R} \times$ $(-C / \varepsilon, C / \varepsilon)=X_{0}^{-1}\left(\mathfrak{T}_{i}\right)$ however we will see that it is more convenient to find $\phi_{0}$ by solving (4.7) in the whole $\mathbb{R}^{2}$. This is possible since the operator $L_{0}^{*}$ and the right hand side of the equation (4.7) can be naturally understood as defined in $\mathbb{R}^{2}$. Of course at the end only the values of $\phi_{0}$ in the set $N_{0}:=X_{0}^{-1} \operatorname{supp} \eta_{0}$ matter for us. The function $X_{0}^{*} \mathcal{F}$ is defined in the set $\hat{N}_{0}:=X_{0}^{-1} \operatorname{supp} \hat{\eta}_{0}$, which is slightly bigger than $N_{0}$ and in particular intersects with the neighbouring sets $N_{ \pm 1}$. As a consequence $\mathcal{F}$ depends nonlinearly and non locally on the functions $\phi_{0}$ and $h$. Thus, we have

$$
\phi_{ \pm 1}\left(x_{0}, y_{0}\right)=-\phi_{0} \circ X_{0}^{-1}\left(X_{0}\left(x_{0}, y_{0}\right) \mp \mathrm{e}\right), \quad h_{ \pm 1}\left(x_{0}\right)=h_{0} \circ X_{0}^{-1}\left(X_{0}\left(x_{0}, y_{0}\right) \mp \mathrm{e}\right) .
$$

As the last observation we have

Lemma 4.1. The function $X_{0}^{*} \mathcal{F}$ is odd in $\hat{N}_{0}$.

The proof of this lemma follows right away from the properties of the functions $\phi_{i}^{\#}, i=0, \pm 1$ and the definition of the approximate solution $\mathfrak{u}$.

Our task now is to understand the mapping property of the operator $L_{0}^{*}$. For this purpose, we shall recall some known result for operator related to $L_{0}^{*}$. First, we have (see [4]).

Lemma 4.2. Suppose $\theta \in(0, \sqrt{2})$. For each function $f \in \overline{\mathcal{C}}_{-\theta}^{0, \mu}(\mathbb{R})$ satisfying

$$
\int_{\mathbb{R}} f\left(y_{0}\right) H^{\prime}\left(y_{0}\right) d y_{0}=0,
$$

there exists a unique solution $\zeta \in \overline{\mathcal{C}}_{-\theta}^{2, \mu}(\mathbb{R})$ of

$$
-\zeta^{\prime \prime}+\left(3 H^{2}-1\right) \zeta=f,
$$

such that

$$
\int_{\mathbb{R}} \zeta\left(y_{0}\right) H^{\prime}\left(y_{0}\right) d y_{0}=0 .
$$

Moreover,

$$
\|\zeta\|_{\overline{\mathcal{C}}_{-\theta}^{2, \mu}(\mathbb{R})} \leq C\|f\|_{\overline{\mathcal{C}}_{-\theta}^{0, \mu}(\mathbb{R})} .
$$


Second, we consider the operator

$$
\begin{aligned}
\mathbb{L}: \overline{\mathcal{C}}_{\sigma, \theta}^{2, \mu}\left(\mathbb{R}^{2}\right) & \longrightarrow \overline{\mathcal{C}}_{\sigma, \theta}^{0, \mu}\left(\mathbb{R}^{2}\right) \\
\zeta & \longmapsto-\Delta_{\left(x_{0}, y_{0}\right)} \zeta+\left(3 H\left(y_{0}\right)^{2}-1\right) \zeta,
\end{aligned}
$$

where the weighted Hölder spaces above are defined by:

$$
\overline{\mathcal{C}}_{\sigma, \theta}^{l, \mu}\left(\mathbb{R}^{2}\right)=\left(\cosh x_{0}\right)^{\sigma}\left(\cosh y_{0}\right)^{\theta} \mathcal{C}^{l, \mu}\left(\mathbb{R}^{2}\right) .
$$

Proposition 4.1. Given $\theta \in(0, \sqrt{2}), \sigma \in\left(0, \frac{\sqrt{2}}{2}\right)$ with

$$
\theta^{2}+\sigma^{2}<2
$$

Suppose that $f \in \overline{\mathcal{C}}_{-\sigma,-\theta}^{0, \mu}\left(\mathbb{R}^{2}\right)$ satisfies

$$
\int_{\mathbb{R}} f\left(x_{0}, y_{0}\right) H^{\prime}\left(y_{0}\right) d y_{0}=0, \quad \forall x_{0} \in \mathbb{R} .
$$

There exists a unique solution $\zeta \in \overline{\mathcal{C}}_{-\sigma,-\theta}^{2, \mu}\left(\mathbb{R}^{2}\right)$ of $\mathbb{L} \zeta=f$, such that

$$
\int_{\mathbb{R}} \zeta\left(x_{0}, y_{0}\right) H^{\prime}\left(y_{0}\right) d y_{0}=0, \quad \forall x_{0} \in \mathbb{R},
$$

and

$$
\|\zeta\|_{\overline{\mathcal{C}}_{-\sigma,-\theta}^{2, \mu}\left(\mathbb{R}^{2}\right)} \leq C\|f\|_{\overline{\mathcal{C}}_{-\sigma,-\theta}^{0, \mu}\left(\mathbb{R}^{2}\right)},
$$

with a constant $C$ independent of $\sigma$.

Proof. We refer to [5] for a proof of this result.

Now let $\delta \in(0, \sqrt{2})$ and let $\tau>0, \mu \in(0,1)$ be constants to be determined later on, both independent of $\varepsilon$. We will come back to equation (4.7). To solve it, one should study its limit as $x_{0} \rightarrow+\infty$. We define the operator $L_{\infty}$ by

$$
\psi \longmapsto-\psi_{\infty}^{\prime \prime}+\left(3 H_{\infty}^{2}-1\right) \psi_{\infty} .
$$

Here $H_{\infty}\left(y_{0}\right)=H\left(y_{0}-\lim _{x_{0} \rightarrow \infty} h\left(x_{0}\right)\right)$. Next, we denote

$$
\mathcal{K}_{0}:=\lim _{x_{0} \rightarrow \infty}\left(X_{0}^{*} \hat{\eta}_{0}\right) \mathcal{K}_{1},
$$

where

$$
\begin{aligned}
\mathcal{K}_{1}\left(y_{0} ; \psi\right)= & \lim _{x_{0} \rightarrow \infty} X_{0}^{*}(E(\mathfrak{u}))-3 \lim _{x_{0} \rightarrow \infty}\left(X_{0}^{*} \mathfrak{u}\right) \psi^{2}-\psi^{3} \\
& -3 \sum_{i=0, \pm 1} \lim _{x_{0} \rightarrow \infty}\left[X_{0}^{*}\left(\mathfrak{u}^{2}-H_{0}^{2}\right) \eta_{i}\right] \psi \\
& -\sum_{i=0, \pm 1}\left[2 \lim _{x_{0} \rightarrow \infty}\left(X_{0}^{*} \nabla \eta_{i}\right) \nabla \psi+\lim _{x_{0} \rightarrow \infty}\left(X_{0}^{*} \Delta \eta_{i}\right) \psi\right] .
\end{aligned}
$$

Proposition 4.2. Suppose $\|h\|_{\overline{\mathcal{C}}_{-\delta}^{2, \mu}\left(\mathbb{R}_{o}\right) \oplus \mathcal{E}_{o}} \leq \varepsilon^{\beta_{0}}$ (as in (3.1)) and $\varepsilon$ is small. There is a unique bounded solution $\psi_{\infty}$ to the equation

$$
L_{\infty} \psi=\mathcal{K}_{0}-\frac{\int_{\mathbb{R}} \mathcal{K}_{0} H_{\infty}^{\prime}}{\int_{\mathbb{R}} H_{\infty}^{\prime 2}} H_{\infty}^{\prime}
$$

satisfiying $\int_{\mathbb{R}} \psi H_{\infty}^{\prime} d y_{0}=0$. In addition $\psi_{\infty}$ is an odd function and

$$
\left\|\psi_{\infty}\right\|_{\overline{\mathcal{C}}_{-\tau}^{2, \mu}(\mathbb{R})} \leq C \varepsilon^{2-\frac{\tau}{\sqrt{2}}}
$$


Proof. The proof follows from Lemma 4.2 and a fixed point argument as soon as we show that

$$
\left\|\hat{\chi}_{\infty} E_{\infty}\right\|_{\overline{\mathcal{C}}_{-\tau}^{2, \mu}(\mathbb{R})} \leq C \varepsilon^{2-\frac{\tau}{\sqrt{2}}}
$$

where

$$
E_{\infty}=\lim _{x_{0} \rightarrow \infty} X_{0}^{*}(E(\mathfrak{u})), \quad \hat{\chi}_{\infty}=\lim _{x_{0} \rightarrow \infty} X_{0}^{*} \hat{\eta}_{0} .
$$

As this type of estimate can be found in [5] we left the details to the reader.

Evidently, we can use the above Proposition to solve the analogous problem as $x_{0} \rightarrow-\infty$. This amounts to solving the same equation as (4.10) but with the function $\mathcal{K}_{0}$ on the right hand side replaced by its analog $\overline{\mathcal{K}}_{0}$ as $x_{0} \rightarrow-\infty$. Taking the limit $x_{0} \rightarrow-\infty$ in $(4.9)$ and using the oddness of the function $X_{0}^{*} \mathcal{F}$ we see that $\overline{\mathcal{K}}_{0}\left(y_{0}\right)=-\mathcal{K}_{0}\left(-y_{0}\right)$ and thus $\psi_{\infty}\left(y_{0}\right)=-\psi_{-\infty}\left(y_{0}\right)$. To write these two solutions conveniently we introduce an odd, smooth function $\kappa$ such that $\kappa\left(x_{0}\right)=1$ when $x_{0}>0$ is large, $\kappa$ is 0 in a neighbourhood of 0 . Then, we let $\psi:=\psi_{\infty}$ and we agree that $\kappa \psi$ is by definition equal to the solution our problem at infinity. We note that this function depends in addition on the $\lim _{x_{0} \rightarrow \infty} h\left(x_{0}\right)=-\lim _{x_{0} \rightarrow-\infty} h\left(x_{0}\right)$. At this point we will not make this dependence explicit in the notation.

We recall that in $(3.2)$ we have defined the function $H_{0}$ locally in $\mathfrak{T}_{0}$ by

$$
X_{0}^{*} H_{0}^{\prime}\left(x_{0}, y_{0} ; h\right)=H^{\prime}\left(y_{0}-h_{0}\left(\varepsilon x_{0}\right)\right) \text {. }
$$

For a problem involving the operator $L_{0}^{*}$, which is defined for all $\left(x_{0}, y_{0}\right) \in \mathbb{R}^{2}$ it is natural to extend the definition of $H_{0}^{\prime}$ to the whole plane. In the sequel, when we speak of a problem defined in $\mathbb{R}^{2}$ we will not write $X_{0}^{*} H_{0}^{\prime}$ but simply $H_{0}^{\prime}$.

For each $g \in \overline{\mathcal{C}}_{-\varepsilon \delta,-\tau}^{0, \mu}\left(\mathbb{R}^{2}\right)$ which is a function of variables $x_{0}$ and $y_{0}$, we will denote

$$
\pi_{1}(g):=\frac{\int_{\mathbb{R}} g H_{0}^{\prime} d y_{0}}{\int_{\mathbb{R}} H_{0}^{\prime 2} d y_{0}} H_{0}^{\prime},
$$

and

$$
\pi_{2}(g)=g-\pi_{1}(g)
$$

With these notations we have

$$
\int_{\mathbb{R}} \pi_{2}(g) H_{0}^{\prime} d y_{0}=0, \quad \forall x_{0} \in \mathbb{R}
$$

Proposition 4.3. Let $\delta, \tau>0$ with $|\delta|,|\tau|$ small. Suppose $\varepsilon>0$ is small enough. Then there exists function $\mathrm{k}=\mathrm{k}\left(x_{0} ; h\right)$ and a function $\phi_{0}=\phi_{0}\left(x_{0}, y_{0} ; h\right)$ such that

$$
L_{0}^{*} \phi_{0}=X_{0}^{*} \mathcal{F}-\mathrm{k}\left(x_{0}\right) H_{0}^{\prime},
$$

with $\pi_{1}\left(\phi_{0}\right)=0$, and

$$
\left\|\phi_{0}-\kappa\left(x_{0}\right) \psi\right\|_{C_{-\varepsilon \delta,-\tau}^{2, \mu}\left(\mathbb{R}^{2}\right)} \leq C \varepsilon^{2-\frac{\tau}{\sqrt{2}}} .
$$

Proof. We first show the Proposition assuming $h=0$. As in Proposition 4.1 denote the operator

$$
\zeta \longmapsto-\Delta_{\left(x_{0}, y_{0}\right)} \zeta+\left(3 H_{0}^{2}-1\right) \zeta
$$

by $\mathbb{L}$. Let us consider the equation

$$
\mathbb{L} \phi_{0}=\pi_{2} \circ\left(\left(\mathbb{L}-L_{0}^{*}\right) \phi_{0}+X_{0}^{*} \mathcal{F}\right) .
$$

By the results of Proposition 4.1, we can write this equation as

$$
\phi_{0}=\mathcal{J} \circ \pi_{2} \circ\left(\left(\mathbb{L}-L_{0}^{*}\right) \phi_{0}+X_{0}^{*} \mathcal{F}\right),
$$

where $\mathcal{J}$ is a linear operator from $\pi_{2}\left(\overline{\mathcal{C}}_{-\varepsilon \delta,-\tau}^{0, \mu}\left(\mathbb{R}^{2}\right)\right)$ to $\pi_{2}\left(\overline{\mathcal{C}}_{-\varepsilon \delta,-\tau}^{2, \mu}\left(\mathbb{R}^{2}\right)\right)$ and whose inverse is bounded by a constant. Suppose $\phi_{0}=\kappa\left(x_{0}\right) \psi\left(y_{0}\right)+\bar{\phi}_{0}$. Then $\bar{\phi}_{0}$ satisfies

$$
\bar{\phi}_{0}=\mathcal{J} \circ \pi_{2} \circ\left(\left(\mathbb{L}-L_{0}^{*}\right)\left(\kappa \psi+\bar{\phi}_{0}\right)+X_{0}^{*} \mathcal{F}\right)-\kappa \psi .
$$

Keep in mind that in this notation, the function $\mathcal{F}$ actually also depends on $\bar{\phi}_{0}$. 
To solve equation (4.13), we shall use a fixed point argument for $\bar{\phi}_{0}$ in the space

$$
\mathcal{X}:=\left\{\zeta \in \overline{\mathcal{C}}_{-\varepsilon \delta,-\tau}^{2, \mu}\left(\mathbb{R}^{2}\right): \int_{\mathbb{R}} \zeta\left(x_{0}, y_{0}\right) H_{0}^{\prime} d y_{0}=0, \forall x_{0} \in \mathbb{R}\right\} .
$$

We will show that the map $M$ defined by

$$
\bar{\phi}_{0} \longmapsto \mathcal{J} \circ \pi_{2} \circ\left(\left(\mathbb{L}-L_{0}^{*}\right)\left(\kappa \psi+\bar{\phi}_{0}\right)+X_{0}^{*} \mathcal{F}\right)-\kappa \psi
$$

is a contraction map in a ball $B_{\rho}(0)$ of $\mathcal{X}$, where the radius $\rho$ is small (to be chosen).

Lemma 4.3. We have $M(0) \in \overline{\mathcal{C}}_{-\varepsilon \delta,-\tau}^{2, \mu}\left(\mathbb{R}^{2}\right)$.

We give a proof this Lemma in the Appendix.

Continuing with the proof of the proposition let us first check that for $i=0, \pm 1$, the map $\mathcal{M}_{i}$

$$
\bar{\phi}_{0} \longmapsto \mathcal{J} \circ \pi_{2} \circ X_{0}^{*}\left(\hat{\eta}_{0}\left[L_{i}, \eta_{i}\right] \phi_{i}^{\#}\right)
$$

is a contraction. For two functions $\bar{\phi}_{i, 1}$ and $\bar{\phi}_{i, 2}$,

$$
\begin{aligned}
\mathcal{M}_{i} & \left(\bar{\phi}_{i, 1}\right)-\mathcal{M}_{i}\left(\bar{\phi}_{i, 2}\right) \\
& =\mathcal{J} \circ \pi_{2} \circ X_{0}^{*}\left(\hat{\eta}_{0}\left[L_{i}, \eta_{i}\right]\left(\bar{\phi}_{i, 1}^{\#}-\bar{\phi}_{i, 2}^{\#}\right)\right),
\end{aligned}
$$

where

$$
\bar{\phi}_{i, j}^{\#}(z)=\bar{\phi}_{0, j}^{\#}(z-i \mathrm{e})=\bar{\phi}_{0, j}\left(X_{0}\left(x_{0}, y_{0}\right)-i \mathrm{e}\right) .
$$

Observe that $\left[L_{i}, \eta_{i}\right]\left(\bar{\phi}_{i, 1}^{\#}-\bar{\phi}_{i, 2}^{\#}\right)$ is equal to zero in the region where $\eta_{i}$ is equal to 1 or 0 . Hence

$$
\begin{aligned}
& \left\|X_{0}^{*} \hat{\eta}_{0}\left[L_{i}, \eta_{i}\right]\left(\bar{\phi}_{i, 1}^{\#}-\bar{\phi}_{i, 2}^{\#}\right)\right\|_{\overline{\mathcal{C}}_{-\varepsilon \delta,-\tau}^{0, \mu}\left(\mathbb{R}^{2}\right)} \\
& =\left\|\cosh ^{\varepsilon \delta} x_{0} \cosh ^{\tau} y_{0} X_{0}^{*}\left[\hat{\eta}_{0}\left[L_{i}, \eta_{i}\right]\left(\bar{\phi}_{i, 1}^{\#}-\bar{\phi}_{i, 2}^{\#}\right)\right]\right\|_{\mathcal{C}^{0, \mu}\left(\mathbb{R}^{2}\right)} \\
& \leq o(1)\left\|X_{0}^{*}\left[\hat{\eta}_{0}\left(\bar{\phi}_{i, 1}^{\#}-\bar{\phi}_{i, 2}^{\#}\right)\right]\right\|_{\overline{\mathcal{C}}_{-\varepsilon \delta,-\tau}^{2, \mu}\left(\mathbb{R}^{2}\right)} \\
& \leq o(1)\left\|\left(X_{0}^{*} \hat{\eta}_{0}\right)\left(\bar{\phi}_{i, 1}-\bar{\phi}_{i, 2}\right)\right\|_{\overline{\mathcal{C}}_{-\varepsilon \delta,-\tau}^{2, \mu}\left(\mathbb{R}^{2}\right)} .
\end{aligned}
$$

The $o(1)$ factor comes from (3.3) by choosing $\varsigma$ small. Consequently, $\mathcal{M}_{i}$ is a contraction mapping. This and (4.14) in turn implies that the map

$$
\bar{\phi}_{0} \rightarrow \mathcal{J} \circ \pi_{2} \circ X_{0}^{*}\left(\hat{\eta}_{0} \sum_{i \in \mathbb{Z}}\left[L_{i}, \eta_{i}\right] \bar{\phi}_{i}^{\#}\right)
$$

is a contraction.

We proceed to consider the map

$$
\mathcal{N}_{i}: \phi_{0} \rightarrow \mathcal{J} \circ \pi_{2} \circ X_{0}^{*}\left(\hat{\eta}_{0}\left(\mathcal{T}-L_{i}\right)\left(\eta_{i} \bar{\phi}_{i}^{\#}\right)\right) .
$$

When $i=0$, from fact that in the set $\operatorname{supp} \eta_{0}$ we have

$$
H_{0}-\mathfrak{u}=o(1),
$$

we deduce

$$
\left\|X_{0}^{*} \hat{\eta}_{0}\left(\mathcal{T}-L_{0}\right)\left(\eta_{0} \bar{\phi}_{0}^{\#}\right)\right\|_{\overline{\mathcal{C}}_{-\varepsilon \delta,-\tau}^{0, \mu}\left(\mathbb{R}^{2}\right)} \leq o(1)\left\|\bar{\phi}_{0}\right\|_{\overline{\mathcal{C}}_{-\varepsilon \delta, \mu}^{0, \mu}\left(\mathbb{R}^{2}\right)} .
$$

Likewise, for $i= \pm 1$, we use the fact that in the set $\operatorname{supp} \hat{\eta}_{0} \cap \operatorname{supp} \eta_{i}$ we have

$$
H_{i}-\mathfrak{u}=o(1),
$$

to conclude

$$
\left\|X_{0}^{*} \bar{\eta}_{0}\left(\mathcal{T}-L_{i}\right)\left(\eta_{i} \bar{\phi}_{1}^{\#}\right)\right\|_{\overline{\mathcal{C}}_{-\varepsilon \delta,-\tau}^{0, \mu}\left(\mathbb{R}^{2}\right)} \leq o(1)\left\|\bar{\phi}_{0}\right\|_{\overline{\mathcal{C}}_{-\varepsilon \delta, \mu}^{0, \mu}\left(\mathbb{R}^{2}\right)} .
$$


In fact, a similar argument shows that $\mathcal{N}_{i}$ are contractions.

We also need to investigate the term

$$
\mathcal{J} \circ \pi_{2} \circ X_{0}^{*}\left[\hat{\eta}_{0}(E(\mathfrak{u})-P(\mathfrak{u}, \bar{\phi}+\kappa \psi))\right] .
$$

Recalling that $P(\mathfrak{u}, \varphi)=3 \mathfrak{u} \varphi^{2}+\varphi^{3}$ we see that it is not difficult to show that the map

$$
\bar{\phi}_{0} \rightarrow \mathcal{J} \circ \pi_{2} \circ X_{0}^{*}\left(\eta_{0} P(\mathfrak{u}, \bar{\phi}+\kappa \psi)\right)
$$

is a contraction if $\left\|\bar{\phi}_{0}\right\|_{\overline{\mathcal{C}}_{-\varepsilon \delta,-\tau}^{0, \mu}\left(\mathbb{R}^{2}\right)}$ is small.

On the other hand, using the expression (4.1) for the Laplacian operator, we infer that for $\varepsilon$ small, the map

$$
\bar{\phi}_{0} \rightarrow \mathcal{J} \circ \pi_{2} \circ\left(\mathbb{L}-L_{0}^{*}\right) \bar{\phi}_{0}
$$

is also a contraction map in a small ball of $\overline{\mathcal{C}}_{-\varepsilon \delta,-\tau}^{0, \mu}\left(\mathbb{R}^{2}\right)$.

Combining all these estimates, we conclude that in the ball of radius

$$
2\left\|\mathcal{J} \circ \pi_{2} \circ X_{0}^{*}\left(\eta_{0} E(\mathfrak{u})\right)\right\|_{\mathcal{C}_{-\varepsilon \delta,-\tau}^{0, \mu}\left(\mathbb{R}^{2}\right)} \leq C \varepsilon^{2-\frac{\tau}{\sqrt{2}}},
$$

of the space $\mathcal{X}$, we get a unique fixed point $\bar{\phi}_{0}$. The corresponding function $\phi_{0}$ and the function $\mathrm{k}$ given by

$$
\mathrm{k}=\pi_{1}\left(\left(\mathbb{L}-L_{0}^{*}\right) \phi_{0}+X_{0}^{*} \mathcal{F}\right) .
$$

satisfy the estimates stated in the proposition.

In the case $h \neq 0$, we let

$$
\phi_{0}\left(x_{0}, y_{0}\right)=\kappa\left(x_{0}\right) \psi\left(y_{0}-h\left(\varepsilon x_{0}\right)\right)+\bar{\phi}_{0}\left(x_{0}, y_{0}\right)
$$

and introduce a change of variables:

$$
\left\{\begin{array}{l}
\tilde{x}_{0}=x_{0} \\
\tilde{y}_{0}=y_{0}-h\left(\varepsilon x_{0}\right)
\end{array}\right.
$$

Then we get a similar equation as (4.13) up to some additional terms involving $h$, which could be treated as perturbation terms.

4.2. The reduced equation and Proof of Theorem 1. For each function $h$ with $\|h\|_{\overline{\mathcal{C}}_{-\delta}^{2, \mu}\left(\mathbb{R}_{o}\right) \oplus \mathcal{E}_{o}} \leq \varepsilon^{\beta_{0}}$, we have obtained $\phi_{0}=\phi_{0}(\cdot ; h)$, which is a function of $x_{0}, y_{0}$, and $\mathrm{k}=\mathrm{k}(\cdot ; h)$, which is a function of $x_{0}$, solving the equation (4.11). In this section we will find an $h$ such that the corresponding $\mathrm{k}$ is identically zero. From this and (4.11), it will follow that the corresponding function $\mathfrak{u}+\phi$ is a solution of (1.1) which satisfies the assertions of Theorem 1.1 and thus we will complete its proof.

By definition we have

$$
\mathrm{k}\left(x_{0}\right)=\pi_{1}\left(L_{0}^{*} \phi_{0}-X_{0}^{*} \mathcal{F}\right) .
$$

Hence to find a $h$ such that $\mathrm{k}=0$, it remains to find $\phi_{0}=\phi_{0}(\cdot ; h)$ satisfying

$$
\pi_{1}\left(L_{0}^{*} \phi_{0}-X_{0}^{*} \mathcal{F}\right)=0 \Longrightarrow \int_{\mathbb{R}} L_{0}^{*} \phi_{0} H_{0}^{\prime} d y_{0}=\int_{\mathbb{R}} X_{0}^{*} H_{0}^{\prime} d y_{0}
$$

Let us investigate the left hand side of (4.15). Recall that one term contained in $\mathcal{F}$ is $\hat{\eta}_{0} E(\mathfrak{u})$, this term is of most interest for us. For notational simplicity, we shall denote

$$
\tilde{h}_{i}(x)=h_{i}(\varepsilon x)
$$

(recall that $h_{i}(x)=h\left(x-i \alpha^{-1}\right)$ ). We will use $\beta$ as a universal positive constant, which may change from step to step. In addition when we choose $\tau$ sufficiently small we mean that at least $2\left(2-\frac{\tau}{\sqrt{2}}\right)>2+\beta^{*}$, with some $\beta^{*}>0$, small and fixed. 
Lemma 4.4. There exists $\bar{\beta}>0$, independent of $\varepsilon, \delta, \tau, \mu, \beta_{0}$, such that for $\varepsilon, \tau$ sufficiently small we have

$$
\begin{aligned}
\int_{\mathbb{R}} X_{0}^{*}\left(\hat{\eta}_{0} E(\mathfrak{u})\right) H_{0}^{\prime} d y_{0} & =c_{*} \tilde{h}_{0}^{\prime \prime}+\sqrt{2} e^{\sqrt{2}\left(q_{0}-q_{1}\right)}\left(\tilde{h}_{0}-\tilde{h}_{1}\right)- \\
& \sqrt{2} e^{\sqrt{2}\left(q_{-1}-q_{0}\right)}\left(\tilde{h}_{-1}-\tilde{h}_{0}\right) \\
& +\mathcal{O}_{\overline{\mathcal{C}}_{-\varepsilon \delta}^{2, \mu}\left(\mathbb{R}_{o}\right) \oplus \mathcal{E}_{o}}\left(\varepsilon^{2+\min \left\{2 \beta_{0}, \bar{\beta}\right\}}\right),
\end{aligned}
$$

for some positive constant $c_{*}$.

Proof. This follows from similar computation as that of [5], we omit the details.

By $\beta$, we will denote a universal positive constant. Making use of Lemma 4.4, we now prove:

Proposition 4.4. There exists $\beta_{1}>0$, independent of $\varepsilon, \delta, \tau, \mu, \beta_{0}$, such that for all $\varepsilon, \tau$ sufficiently small, equation (4.15) can be written as

$$
c_{*}^{\prime \prime} \tilde{h}_{0}+\sqrt{2} e^{\sqrt{2}\left(q_{0}-q_{1}\right)}\left(\tilde{h}_{0}-\tilde{h}_{1}\right)-\sqrt{2} e^{\sqrt{2}\left(q_{-1}-q_{0}\right)}\left(\tilde{h}_{-1}-\tilde{h}_{0}\right)=G(\cdot ; \tilde{h}),
$$

where the function $G$ satisfies

$$
\|G(\cdot ; 0)\|_{\overline{\mathcal{C}}_{-\varepsilon \delta}^{0, \mu}\left(\mathbb{R}_{o}\right)} \leq C \varepsilon^{2+\beta_{1}} .
$$

Moreover, for any two functions $h^{\sharp}, h^{b}$ with

$$
\begin{aligned}
& \left\|h^{\sharp}\right\|_{\overline{\mathcal{C}}_{-\delta}^{2, \mu}\left(\mathbb{R}_{o}\right) \oplus \mathcal{E}_{o}} \leq \varepsilon^{\beta_{0}}, \\
& \left\|h^{b}\right\|_{\overline{\mathcal{C}}_{-\delta}^{2, \mu}\left(\mathbb{R}_{o}\right) \oplus \mathcal{E}_{o}} \leq \varepsilon^{\beta_{0}},
\end{aligned}
$$

there holds

$$
\left\|G\left(\cdot ; h^{\sharp}(\varepsilon \cdot)\right)-G\left(\cdot ; h^{b}(\varepsilon \cdot)\right)\right\|_{\overline{\mathcal{C}}_{-\varepsilon \delta}^{2, \mu}\left(\mathbb{R}_{o}\right)} \leq C \varepsilon^{2+\min \left\{2 \beta_{0}, \beta_{1}\right\}}\left(\left\|h^{\sharp}-h^{b}\right\|_{\overline{\mathcal{C}}_{-\delta}^{2, \mu}\left(\mathbb{R}_{o}\right) \oplus \mathcal{E}_{o}}\right) .
$$

Proof. Essentially, this follows from the estimates of $\phi_{0}$ obtained in Proposition 4.3 and similar computation as that of Lemma 4.4. We sketch the proof and refer to [5] for more details.

First of all, let us consider the term $\int_{\mathbb{R}} L_{0}^{*} \phi_{0} H_{0}^{\prime} d y_{0}$. We have

$$
\begin{aligned}
\int_{\mathbb{R}} L_{0}^{*} \phi_{0} H_{0}^{\prime} d y_{0} & =\int_{\mathbb{R}} X_{0}^{*}\left[\left(-\Delta \phi_{0}^{\#}+\left(3 H_{0}^{2}-1\right) \phi_{0}^{\#}\right)\right] H_{0}^{\prime} d y_{0} \\
& =\underbrace{\int_{\mathbb{R}}\left(-\partial_{y_{0}}^{2} \phi_{0}+\left(3 X_{0}^{*} H_{0}^{2}-1\right) \phi_{0}\right) H_{0}^{\prime} d y_{0}}_{I} \\
& +\underbrace{\int_{\mathbb{R}}\left(-\partial_{x_{0}}^{2} \phi_{0}\right) H_{0}^{\prime} d y_{0}}_{I I}+\underbrace{\int_{\mathbb{R}}\left(\Delta_{\left(x_{0}, y_{0}\right)} \phi_{0}-X_{0}^{*} \Delta \phi_{0}^{\#}\right) H_{0}^{\prime} d y_{0}}_{I I I} .
\end{aligned}
$$

Integration by parts shows that $I=0$. On the other hand, using the estimate for $\phi_{0}$ and $h$ and the fact that

$$
\int_{\mathbb{R}} \phi_{0} H_{0}^{\prime} d y_{0}=0
$$

we see that

hence

$$
I I=2 \int_{\mathbb{R}} \partial_{x_{0}} \phi_{0} \partial_{x_{0}} H_{0}^{\prime} d y_{0}+\int_{\mathbb{R}} \phi_{0} \partial_{x_{0}}^{2} H_{0}^{\prime} d y_{0},
$$

$$
\|I I\|_{\overline{\mathcal{C}}_{-\varepsilon \delta}^{2, \mu}\left(\mathbb{R}_{o}\right)} \leq C \varepsilon^{2+\beta}
$$

Furthermore, by the expression of the Laplacian in the Fermi coordinate, the last integral is of the order $\mathcal{O}_{\overline{\mathcal{C}}_{-\varepsilon \delta}^{2, \mu}\left(\mathbb{R}_{o}\right)}\left(\varepsilon^{2+\beta}\right)$. 
Next, we will analyze the term $\int_{\mathbb{R}} X_{0}^{*} \mathcal{F} H_{0}^{\prime} d y_{0}$. By Proposition 4.3,

$$
\phi_{0}\left(x_{0}, y_{0}\right)=\kappa\left(x_{0}\right) \psi\left(y_{0}-h\left(\varepsilon x_{0}\right)\right)+\mathcal{O}_{\overline{\mathcal{C}}_{-\varepsilon \delta,-\tau}^{2, \mu}\left(\mathbb{R}^{2}\right)}\left(\varepsilon^{2-\frac{\tau}{\sqrt{2}}}\right) .
$$

Using this one can prove the following:

Lemma 4.5. We have

$$
\int_{\mathbb{R}} X_{0}^{*} \mathcal{F} H_{0}^{\prime} d y_{0}=\int_{\mathbb{R}} X_{0}^{*}\left(\hat{\eta}_{0} E(\mathfrak{u})\right) H_{0}^{\prime} d y_{0}+\mathcal{O}_{\overline{\mathcal{C}}_{-\varepsilon \delta}^{2, \mu}(\mathbb{R})}\left(\varepsilon^{2+\beta}\right) .
$$

The proof of this lemma is left to the reader.

The equation (4.4) then follows from the above estimates and Lemma 4.4. The proof of the fact that $G(\cdot ; h)$ is Lipschitz is similar to that of $[5]$, we omit the details.

With all these preparations at hand, we are in a position to prove the main theorem.

Proof of Theorem 1.1. By Proposition 4.4 the linearized operator $\mathcal{L}_{1}$ of the one-soliton to the Toda lattice is an isomorphism from $\overline{\mathcal{C}}_{-\delta}^{2, \mu}\left(\mathbb{R}_{o}\right) \oplus \mathcal{E}_{o}$ into $\overline{\mathcal{C}}_{-\delta}^{0, \mu}\left(\mathbb{R}_{o}\right)$. With the notation of Proposition 4.4, the projected equation (4.15) can be written as

$$
h(\cdot)=\varepsilon^{-2} \mathcal{L}_{1}^{-1} \circ G\left(\frac{\dot{\varepsilon}}{\varepsilon} ; h\right) .
$$

Using Proposition 4.4 and Proposition 2.4, if we choose $\tau$ sufficiently small independent of $\varepsilon$, and $\mu=$ $\frac{\beta_{1}}{4}, \beta_{0}=\frac{\beta_{1}}{2}$, then the map given by

$$
h \longmapsto \varepsilon^{-2} \mathcal{L}_{1}^{-1} \circ G\left(\frac{\dot{-}}{\varepsilon} ; h\right)
$$

will be a contraction in the ball of radius $\varepsilon^{\beta_{0}}$ in $\overline{\mathcal{C}}_{-\delta}^{2, \mu}\left(\mathbb{R}_{o}\right) \oplus \mathcal{E}_{o}$. Indeed, it is straightforward to check that

$$
\left\|G\left(\frac{\dot{r}}{\varepsilon} ; h\right)\right\|_{\overline{\mathcal{C}}_{-\delta}^{0, \mu}\left(\mathbb{R}_{o}\right)} \leq C \varepsilon^{-\mu}\|G(\cdot ; h(\varepsilon \cdot))\|_{\overline{\mathcal{C}}_{-\varepsilon \delta}^{0, \mu}\left(\mathbb{R}_{o}\right)} \leq C \varepsilon^{2+\min \left\{2 \beta_{0}, \beta_{1}\right\}-\mu}\|h\|_{\overline{\mathcal{C}}_{-\delta}^{0, \mu}\left(\mathbb{R}_{o}\right)},
$$

where we have used of Proposition 4.4. By a standard fixed point argument we find an $h$ with $\|h\|_{\overline{\mathcal{C}}_{-\delta}^{2, \mu}\left(\mathbb{R}_{o}\right) \oplus \mathcal{E}_{o}} \leq$ $\varepsilon^{\beta_{0}}$. This completes the proof of the theorem.

\section{APPENDIX}

Proof of Lemma 4.3. Recall that we assume here $h=0$. Denoting the inverse of the operator $L_{\infty}$ (see (4.8)) restricted to a subspace of $\mathcal{X}$ consisting of functions which are $x_{0}$ independent by $\mathcal{J}_{\infty}$ we can write:

$$
M(0)=\mathcal{J} \circ \pi_{2} \circ\left(\left(\mathbb{L}-L_{0}^{*}\right) \kappa \psi+X_{0}^{*} \mathcal{F}\right)-\mathcal{J}_{\infty} \circ \pi_{2} \circ\left(\kappa \mathcal{K}_{0}\right) .
$$

By definition of the operators $\mathbb{L}$ and $L_{0}^{*}$ and formula (4.1) it is easy to see that

$$
\mathcal{J} \circ \pi_{2} \circ\left(\left(\mathbb{L}-L_{0}^{*}\right) \kappa \psi\right) \in \overline{\mathcal{C}}_{-\varepsilon \delta,-\tau}^{2, \mu}\left(\mathbb{R}^{2}\right) .
$$

It remains to estimate

$$
\mathcal{J} \circ \pi_{2} \circ X_{0}^{*} \mathcal{F}-\mathcal{J}_{\infty} \circ \pi_{2} \circ\left(\kappa \mathcal{K}_{0}\right)=\underbrace{\mathcal{J} \circ \pi_{2} \circ\left(X_{0}^{*} \mathcal{F}-\kappa \mathcal{K}_{0}\right)}_{A_{1}}+\underbrace{\left(\mathcal{J}-\mathcal{J}_{\infty}\right) \circ \pi_{2} \circ\left(\kappa \mathcal{K}_{0}\right)}_{A_{2}} .
$$

Recalling that $\mathcal{K}_{0}=\lim _{x_{0} \rightarrow \infty} X_{0}^{*} \mathcal{F}$ we see that to estimate $A_{1}$ we need to study

$$
X_{0}^{*} \mathcal{F}-\kappa\left(x_{0}\right) \lim _{x_{0} \rightarrow \infty} X_{0}^{*} \mathcal{F} .
$$

The leading order term in this difference is the one involving the error term $E(\mathfrak{u})$. We claim that for this term we have

$$
X_{0}^{*} \hat{\eta}_{0} E(\mathfrak{u})-\kappa\left(x_{0}\right) \lim _{x_{0} \rightarrow \infty} X_{0}^{*} \hat{\eta}_{0} E(\mathfrak{u}) \in \overline{\mathcal{C}}_{-\varepsilon \delta,-\tau}^{2, \mu}\left(\mathbb{R}^{2}\right) .
$$

This is quite easy to see observing that

$$
X_{0}^{*} \hat{\eta}_{0}-\lim _{x_{0} \rightarrow \infty} X_{0}^{*} \hat{\eta}_{0}
$$


decays exponentially in $x_{0}$, which is due to the fact that the lines $q_{i}(x)$ are parallel at infinity up to an exponentially small term. To estimate $A_{2}$ it suffices to estimate

$$
\left(\mathbb{L}-L_{\infty}\right) \kappa\left(x_{0}\right) \psi\left(y_{0}\right) .
$$

But since

it is obvious that

$$
\left(\mathbb{L}-L_{\infty}\right)=-\partial_{x_{0}}^{2}
$$

$$
\left(\mathbb{L}-L_{\infty}\right) \kappa \psi \in \overline{\mathcal{C}}_{-\varepsilon \delta,-\tau}^{0, \mu}\left(\mathbb{R}^{2}\right),
$$

from which we find $A_{2} \in \overline{\mathcal{C}}_{-\varepsilon \delta,-\tau}^{2, \mu}\left(\mathbb{R}^{2}\right)$.

\section{REFERENCES}

[1] F. Alessio, A. Calamai, and P. Montecchiari. Saddle-type solutions for a class of semilinear elliptic equations. Adv. Differential Equations, 12(4):361-380, 2007.

[2] L. Ambrosio and X. Cabré. Entire solutions of semilinear elliptic equations in $\mathbf{R}^{3}$ and a conjecture of De Giorgi. J. Amer. Math. Soc., 13(4):725-739 (electronic), 2000.

[3] H. Dang, P. C. Fife, and L. A. Peletier. Saddle solutions of the bistable diffusion equation. Z. Angew. Math. Phys., 43(6):984998, 1992.

[4] M. del Pino, M. Kowalczyk, and F. Pacard. Moduli space theory for the Allen-Cahn equation in the plane. Trans. Amer. Math. Soc., 365(2):721-766, 2013.

[5] M. del Pino, M. Kowalczyk, F. Pacard, and J. Wei. Multiple-end solutions to the Allen-Cahn equation in $\mathbb{R}^{2}$. J. Funct. Anal., 258(2):458-503, 2010.

[6] M. del Pino, M. Kowalczyk, and J. Wei. On De Giorgi's conjecture in dimension N $\geq$ 9. Ann. of Math. (2), 174(3):1485-1569, 2011.

[7] G. Friesecke and R. L. Pego. Solitary waves on FPU lattices. I. Qualitative properties, renormalization and continuum limit. Nonlinearity, 12(6):1601-1627, 1999.

[8] G. Friesecke and R. L. Pego. Solitary waves on FPU lattices. II. Linear implies nonlinear stability. Nonlinearity, 15(4):1343$1359,2002$.

[9] G. Friesecke and R. L. Pego. Solitary waves on Fermi-Pasta-Ulam lattices. III. Howland-type Floquet theory. Nonlinearity, 17(1):207-227, 2004.

[10] G. Friesecke and R. L. Pego. Solitary waves on Fermi-Pasta-Ulam lattices. IV. Proof of stability at low energy. Nonlinearity, 17(1):229-251, 2004.

[11] N. Ghoussoub and C. Gui. On a conjecture of De Giorgi and some related problems. Math. Ann., 311(3):481-491, 1998.

[12] C. Gui. Symmetry of some entire solutions to the Allen-Cahn equation in two dimensions. J. Differential Equations, 252(11):5853-5874, 2012.

[13] M. Kowalczyk and Y. Liu. Nondegeneracy of the saddle solution of the Allen-Cahn equation. Proc. Amer. Math. Soc., 139(12):4319-4329, 2011.

[14] M. Kowalczyk, Y. Liu, and F. Pacard. The classification of four ended solutions to the Allen-Cahn equation on the plane. to appear Analysis and PDE, 2011.

[15] M. Kowalczyk, Y. Liu, and F. Pacard. The space of 4-ended solutions to the Allen-Cahn equation in the plane. Ann. Inst. H. Poincaré Anal. Non Linéaire, 29(5):761-781, 2012.

[16] M. Kowalczyk, Y. Liu, F. Pacard, and J. Wei. End-to-end construction for the Allen-Cahn equation on the plane. to appear Calculus of Variations and PDE, 2013.

[17] R. Mazzeo, D. Pollack, and K. Uhlenbeck. Moduli spaces of singular Yamabe metrics. J. Amer. Math. Soc., 9(2):303-344, 1996.

[18] T. Mizumachi and R. L. Pego. Asymptotic stability of Toda lattice solitons. Nonlinearity, 21(9):2099-2111, 2008.

[19] F. Pacard. Connected sum contruction in geometry and nonlinear analysis. Unpublished lecture notes, 2008.

[20] O. Savin. Regularity of flat level sets in phase transitions. Ann. of Math. (2), 169(1):41-78, 2009.

[21] J. Shi. Semilinear Neumann boundary value problems on a rectangle. Trans. Amer. Math. Soc., 354(8):3117-3154 (electronic), 2002.

Michą Kowalczyk - Departamento de Ingeniería Matemática and Centro de Modelamiento Matemático (Umi 2807 CNRS), Universidad de Chile, Casilla 170 Correo 3, Santiago, Chile.

E-mail address: kowalczy@dim.uchile.cl

Yong Liu - Department of Mathematics and Physics, North China Electric Power University, Beijing, China.

E-mail address: liuyong@ncepu.edu.cn

Juncheng Wei -Department of Mathematics, University of British Columbia, Vancouver, B.C., Canada V6T 1 Z2 and Department of Mathematics, Chinese University of Hong Kong, Shatin, Hong Kong.

E-mail address: jcwei@math.ubc.ca 